\title{
Enlarging the functional space of decay estimates on semigroups
}

\author{
C. MOUHOT $^{*}$ \\ CNRS \& DMA, ÉNS Paris, \\ 45, rue d'Ulm, F-75230 Paris cedex 05 \\ *E-mail: Clement.Mouhot@ens.fr
}

\begin{abstract}
This note briefly presents a new method for enlarging the functional space of a "spectral-gap-like" estimate of exponential decay on a semigroup. A particular case of the method was first devised in Ref. 1 for the spatially homogeneous Boltzmann equation, and a variant was used in Ref. 2 in the same context for inelastic collisions. We present a generalized abstract version of it, a short proof of the algebraic core of the method, and a new application to the FokkerPlanck equation. More details and other applications shall be found in the work in preparation Ref. 3 (another application to quantum kinetic theory can be found in the work in preparation Ref. 4).
\end{abstract}

Keywords: spectral gap; exponential decay; semigroup; Fokker-Planck equation; Poincaré inequality.

\section{The "space enlargement" issue}

Consider a Hilbert space $\mathcal{H}$, a (possibly unbounded) linear operator $\mathcal{T}$ on $\mathcal{H}$ which generates a strongly continuous semigroup $e^{t \mathcal{T}}$ with spectrum $\Sigma(\mathcal{T})$. Assume that for some Hilbert subspace $H \subset \mathcal{H}$ the restricted operator $T:=\left.\mathcal{T}\right|_{H}$ generates a strongly continuous semigroup $e^{t T}$ with spectrum $\Sigma(T)$ in $H$.

Assume some "spectral-gap-like" information on $\Sigma(T)$, typically when $T$ is self-adjoint assume

$$
\forall f \in H, f \perp \operatorname{Null}(T), \quad\left\|e^{t T} f\right\|_{H} \leq e^{\lambda t}\|f\|_{H}, \quad \lambda<0 .
$$

An important class of applications is the following: $\mathcal{T}$ is a partial differential operator (acting on a large class of function on $\mathbb{R}^{d}$, say $L^{1}$ ), with equilibrium $\mu$ and detailed spectral information available in a much smaller space $H=L^{2}\left(\mu^{-1}\right)$ where it is symmetric. The latter space is much smaller than $\mathcal{H}$ in the sense that it requires a stronger decay condition, e.g. when $\mu$ is a gaussian in statistical mechanics. 
The question addressed here is: can one deduce from the spectral-gap information in the space $H$ some spectral-gap information in the larger space $\mathcal{H}$, and if possible in a quantitative way? More explicitly, does $e^{t} \mathcal{T}$ have the same decay property as $e^{t T}$ above?

We give a positive answer for a class of operators $\mathcal{T}$ which split into a part $\mathcal{A}$ "regularizing" $\mathcal{H}$ into $H$ and a coercive part $\mathcal{B}$. We then show that, under some assumption on the potential force, the Fokker-Planck equation belongs to this class and, as a consequence, we prove that its spectral gap property can be extended from the linearization space (with gaussian decay) to larger $L^{2}$ spaces with, say, polynomial weights.

\section{The abstract result}

Let us start with an almost equivalent condition for the decay of the semigroup in terms of a uniform bound on a vertical line for the resolvent. We omit the proof to keep this note short. It can be found in Ref. 3 and it mainly relies on a careful use of the Parseval identity between the resolvent operator and the semigroup.

For some closed densely defined unbounded operator $T$ in a Hilbert space $E$, denote by by $R(z)=(T-z)^{-1}, z \notin \Sigma(T)$ its resolvent operator, and $\mathcal{L}(E)$ the space of bounded linear operators on $E$. Finally for any $a \in \mathbb{R}$, define the half complex plane $\Delta_{a}:=\{z \in \mathbb{C}$, 凡e $z>a\}$.

Theorem 2.1. Assume for the operator $T$ in the Hilbert space E:

(H1) Localization of the spectrum: $\Sigma(T) \subset\left(\overline{\Delta_{a}}\right)^{c} \cup\left\{\xi_{1}, \ldots, \xi_{k}\right\}$ with $a \in \mathbb{R}$, and $\xi_{j} \in \Delta_{a}, 1 \leq j \leq k$ some discrete eigenvalues;

(H2) Control on the resolvent operators:

$$
\exists K>0, \quad \forall y \in \mathbb{R}, \quad\|R(a+i y)\|_{\mathcal{L}(E)} \leq K .
$$

(H3) Weak control on the semigroup: There exist $b, C_{b} \geq 0$ such that

$$
\forall t \geq 0 \quad\left\|e^{t T}\right\|_{\mathcal{L}(E)} \leq C_{b} e^{b t} .
$$

Then, for any $\lambda>a$, there exists $C_{\lambda}$ explicit from $a, b, C_{b}, K$ such that

$$
\forall t \geq 0, \quad\left\|e^{t T}-\sum_{i=1}^{k} e^{\xi_{i} t} \Pi_{i}\right\|_{\mathcal{L}(E)} \leq C_{\lambda} e^{\lambda t}
$$

for the spectral projectors $\Pi_{i}$ of eigenvalues $\xi_{i}$. 
We also have the following converse result: assume

$$
\forall t \geq 0, \quad\left\|e^{t T}-\sum_{j=1}^{k} e^{\xi_{j} t} \Pi_{j}\right\|_{\mathcal{L}(E)} \leq C_{a} e^{a t}
$$

for some constants $a \in \mathbb{R}, C_{a} \in(0, \infty)$, some complex numbers $\xi_{j} \in \Delta_{a}$ and some operators $\Pi_{j}$ which all commute with $e^{t T}$. Then $T$ satisfies $(\boldsymbol{H 1})$, (H2), (H3).

Remark 2.2. Assumption (H3) is required in this theorem in order to obtain quantitative constants in the rate of decay. Therefore, under assumptions (H1) and (H3), assertions (H2) and Eq. (1) are equivalent in a quantitative way.

The following theorem is the core of the method:

Theorem 2.3. Assume that $\mathcal{T}$ is a closed unbounded densely defined operator in a Hilbert space $\mathcal{H}$, and that $T:=\left.\mathcal{T}\right|_{H}$ is a closed unbounded densely defined operator in a Hilbert subspace $H \subset \mathcal{H}$ which satisfies (H1) and (H2) (with $E=H$ ). Assume moreover that $\mathcal{T}$ satisfies:

(H4) Decomposition: $\mathcal{T}=\mathcal{A}+\mathcal{B}$ where $\mathcal{A}$ and $\mathcal{B}$ are closed unbounded densely defined operators with domains included in the one of $\mathcal{T}$ such that

- for some $r>0$, the operator $\mathcal{B}-\xi$ is invertible with uniform bound for any $\xi \in \Delta_{a} \backslash\left(\cup_{i=1}^{k} B\left(\xi_{i}, r\right)\right.$ ) (where every balls $B\left(\xi_{i}, r\right)$ are strictly included in $\left.\Delta_{a}\right)$;

- $B=\mathcal{B}_{\mid H}$ is well-defined as a closed unbounded densely defined operator with domain included in the one of $T$, and $B-\xi$ is invertible for any $\xi \in \Delta_{a} \backslash\left(\cup_{i=1}^{k} B\left(\xi_{i}, r\right)\right)$;

- $\mathcal{A}(\mathcal{B}-\xi)^{-1}: \mathcal{H} \rightarrow H$ and $(\mathcal{B}-\xi)^{-1} \mathcal{A}: \mathcal{H} \rightarrow H$ are bounded for any $\xi \in \Delta_{a} \backslash\left(\cup_{i=1}^{k} B\left(\xi_{i}, r\right)\right)$.

Then $\mathcal{T}$ satisfies (H2) in the space $E=\mathcal{H}$ (with constructive bounds in terms of the above assumptions).

The proof of the following corollary is immediate by combining Theorem 2.3 and Theorems 2.1 .

Corollary 2.4. Assume that $T$ satisfies (H1) and (H2) in the space $H$ and $\mathcal{T}$ satisfies $(\boldsymbol{H 3})$ and $\left(\boldsymbol{H}_{4}\right)$ in the space $\mathcal{H}$. Assume moreover that the eigenvalues of $\mathcal{T}$ in $\Delta_{a}$ are the same as those of $T$, that is $\left\{\xi_{1}, \ldots, \xi_{k}\right\}$. 
Then the conclusion of Theorem 2.1 holds in the space $\mathcal{H}$ : for any $\lambda>a$, there exists $C_{\lambda}$ explicit from $a, b, C_{b}, K$ such that

$$
\forall t \geq 0, \quad\left\|e^{t \mathcal{T}}-\sum_{i=1}^{k} e^{\xi_{i} t} \Pi_{i}\right\|_{\mathcal{L}(\mathcal{H})} \leq C_{\lambda} e^{\lambda t} .
$$

Remark 2.5. If $r$ can be taken as small as wanted (H4) (for some decompositions depending on $r$ ), it can be proved that the eigenvalues of $\mathcal{T}$ in $\Delta_{a}$ are the same as those of $T$ in $\Delta_{a}$ (that is $\left\{\xi_{1}, \ldots, \xi_{k}\right\}$ ) and this assumption can be relaxed.

Remark 2.6. Thanks to the reciprocal part of Theorem 2.1, assumption (H2) on $T$ can be replaced by assuming a decay on the semigroup:

$$
\forall t \geq 0, \quad\left\|e^{t T}-\sum_{j=1}^{k} e^{\xi_{j} t} \Pi_{j}\right\|_{L(H)} \leq C_{\lambda} e^{\lambda t} .
$$

Proof of Theorem 2.3. Assume that $k=1$ and $\xi_{1}=0$ for the sake of simplicity, the proof being similar in the general case.

Take $\xi \notin \Delta_{a} \backslash B(0, r)$ and define

$$
U(\xi):=\mathcal{B}(\xi)^{-1}-R(\xi) \mathcal{A B}(\xi)^{-1},
$$

where $R(\xi)$ is the resolvent of $T$ in $H$ and $\mathcal{B}(\xi)=\mathcal{B}-\xi$. Since by assumption $\mathcal{B}(\xi)^{-1}: H \rightarrow H, \mathcal{A B}(\xi)^{-1}: \mathcal{H} \rightarrow H$ and $R(\xi): H \rightarrow H$ are bounded operators, $U(\xi): H \rightarrow H$ is well-defined and bounded from $\mathcal{H}$ to $\mathcal{H}$. Then,

$$
\begin{aligned}
(\mathcal{T}-\xi) U(\xi) & =(\mathcal{A}+\mathcal{B}(\xi)) \mathcal{B}(\xi)^{-1}-(\mathcal{T}-\xi) R(\xi) \mathcal{A B}(\xi)^{-1} \\
& =\mathcal{A B}(\xi)^{-1}+\operatorname{Id}_{\mathcal{H}}-(\mathcal{T}-\xi) R(\xi) \mathcal{A} \mathcal{B}(\xi)^{-1} \\
& =\mathcal{A B}(\xi)^{-1}+\operatorname{Id}_{\mathcal{H}}-\mathcal{A B}(\xi)^{-1}=\operatorname{Id}_{\mathcal{H}}
\end{aligned}
$$

To be more precise, introduce the canonical injection $J: H \rightarrow \mathcal{H}$ and use that $R=J R, \mathcal{A}=J \mathcal{A}, \mathcal{T} J=J T$ to write:

$$
\begin{aligned}
(\mathcal{T}-\xi) R(\xi) \mathcal{A B}(\xi)^{-1} & =(\mathcal{T}-\xi) J R(\xi) \mathcal{A B}(\xi)^{-1}=J(T-\xi) R(\xi) \mathcal{A B}(\xi)^{-1} \\
& =J \operatorname{Id}_{H} \mathcal{A B}(\xi)^{-1}=J \mathcal{A B}(\xi)^{-1}=\mathcal{A B}(\xi)^{-1}
\end{aligned}
$$

The operator $\mathcal{T}-\xi$ is also one-to-one. Indeed, if $g$ satisfies

$$
g \in \operatorname{Dom}(\mathcal{T}), \quad(\mathcal{T}-\xi) g=0,
$$

the decomposition $(\mathbf{H} 4)$ yields

$$
\mathcal{B}(\xi) g=-\mathcal{A} g \in H,
$$


and therefore $g \in \operatorname{Domain}(B) \subset \operatorname{Domain}(T) \subset H$ because $B(\xi)=\mathcal{B}(\xi)_{\mid H}$ is invertible on $H$. We conclude that $g=0$ since $T-\xi$ is one-to-one. As a conclusion, $U(\xi)$ is the inverse of $\mathcal{T}-\xi$ which in turn implies that $\xi \notin \Sigma(\mathcal{T})$ and $\mathcal{R}(\xi)=U(\xi)$ satisfies the announced estimate. This concludes the proof.

\section{Application to the Fokker-Planck equation}

In this section we are concerned with the Fokker-Planck equation

$$
\partial_{t} f=\mathcal{T} f:=\operatorname{div}(\nabla f+E f)
$$

for the real valued density function $f=f(t, x), t \geq 0, x \in \mathbb{R}^{d}$. In this equation $E=E(x) \in \mathbb{R}^{d}$ is a given force field, written as

$$
E=\nabla U+F
$$

where the potential $U: \mathbb{R}^{d} \rightarrow \mathbb{R}$ is such that $\mu(d x)=e^{-U(x)} d x$ is a probability measure satisfying the "Poincaré inequality condition": there exists $\lambda_{P}<0$ such that

$$
-\int|\nabla u|^{2} \mu(d v) \leq \lambda_{P} \int u^{2} \mu(d v) \quad \forall u \in H^{1}\left(\mathbb{R}^{d}\right), \quad \int u d \mu=0,
$$

( $c f$. for instance Refs. 5-7 and the references therein). The additionnal force field $F$ satisfies

$$
\nabla \cdot F=0, \quad \nabla U \cdot F=0, \quad|F| \leq C(1+|\nabla U|) .
$$

Thanks to that structural assumptions we can split $\mathcal{T}$ between a symmetric term and a skew-symmetric term:

$$
\mathcal{T}=\mathcal{T}^{s}+\mathcal{T}^{a s}, \quad \mathcal{T}^{s} f=\operatorname{div}(\nabla f+\nabla U f), \quad \mathcal{T}^{a s} f=\operatorname{div}(F f) .
$$

The operator $\mathcal{T}^{s}$ is symmetric in $H=L^{2}\left(\mu^{-1}\right)$

$$
\left\langle\mathcal{T}^{s} f, g\right\rangle_{H}=-\int \nabla(f / \mu) \cdot \nabla(g / \mu) \mu=\left\langle f, \mathcal{T}^{s} g\right\rangle_{H},
$$

while the operator $\mathcal{T}^{a s}$ is anti-symmetric in $\mathcal{H}=L^{2}\left(m^{-1}\right)$ for any weight function $m^{-1}(v)=\theta(U(x))$, with $\theta: \mathbb{R}_{+} \rightarrow \mathbb{R}_{+}$:

$$
\begin{aligned}
\left(\mathcal{T}^{a s} f, g\right)_{\mathcal{H}} & =\int[(\nabla \cdot F) f+F \cdot \nabla f] g m^{-1}=-\int f \nabla \cdot\left(F g m^{-1}\right) \\
& =-\int f\left[(\nabla \cdot F) g m^{-1}+(F \cdot \nabla g) m^{-1}+g \theta^{\prime}(U)(\nabla U \cdot F)\right] \\
& =-\int[(\nabla \cdot F) g+F \cdot \nabla g] f m^{-1}=-\left\langle f, \mathcal{T}^{a s} g\right\rangle_{\mathcal{H}} .
\end{aligned}
$$


As an important consequence, we have

$$
\left\langle\mathcal{T}^{a s} f, f\right\rangle_{\mathcal{H}}=\int \nabla \cdot(F f) f \theta(U)=0 .
$$

In $H:=L^{2}\left(\mu^{-1}\right)$ the restricted operator $T:=\left.\mathcal{T}\right|_{H}$ is non-positive, its first eigenvalue is 0 associated to the eigenspace $\mathbb{R} \mu$, and it has a spectral gap thanks to the Poincaré inequality:

$$
\left.\int f(T f) \mu^{-1}=-\int \mu \mid \nabla(f / \mu)\right)\left.\right|^{2} \leq \lambda_{2}\|f-\langle f\rangle\|_{H}^{2} .
$$

A natural question to ask is whether it is possible to obtain an exponential decay on the semigroup in a space larger than $H$. The following theorem gives an answer in $L^{2}$ spaces with polynomial or "stretched" exponential weights. The proof follows from the application of the abstract method and some careful computations on the Dirichlet form in the larger space.

Theorem 3.7. Let $\mu=e^{-U}$ with $U(v)=\left(1+|x|^{2}\right)^{s / 2}, s \geq 1$ (so that Poincaré inequality holds for $\mu$ ).

Let $m \in C^{2}\left(\mathbb{R}^{d}\right)$ be a weight function such that $m^{-1}(x)=\theta(U(x))$ with $\theta(x)=\left(1+|x|^{2}\right)^{k / 2}$ with $k>d$ or $\theta(x)=e^{\left(1+|x|^{2}\right)^{k / 2}}$ with $k \in(0,1)$. Let us define $\mathcal{H}:=L^{2}\left(m^{-1}\right)$.

Then there exist explicit $\lambda \in\left(-\lambda_{P}, 0\right)$ and $C_{\lambda} \in[1, \infty)$ such that

$$
\forall f_{0} \in \mathcal{H}, \quad \forall t \geq 0 \quad\left\|f_{t}-\left\langle f_{0}\right\rangle \mu\right\|_{\mathcal{H}} \leq C_{\lambda} e^{\lambda t}\left\|f_{0}-\left\langle f_{0}\right\rangle \mu\right\|_{\mathcal{H}} .
$$

Remark 3.8. In this theorem $C_{\lambda}>1$ is allowed, which means that we do not prove that the Dirichlet form of $\mathcal{T}$ has a sign.

Remark 3.9. The smoothness assumption on $U$ and $m$ at the origin can be relaxed.

Acknowledgements. The author thanks Stéphane Mischler for useful discussion during the preparation of this note. He also wishes to thank the Award No. KUK-I1-007-43, funded by the King Abdullah University of Science and Technology (KAUST) for the funding provided for his repeated visits at Cambridge University.

\section{References}

1. C. Mouhot, Comm. Math. Phys. 261, 629 (2006).

2. S. Mischler and C. Mouhot, Comm. Math. Phys. 288, 431 (2009).

3. M. Gualdani, S. Mischler and C. Mouhot, Spectral gap in small and large functional spaces, In progress. 
4. A. Arnold, I. Gambda, M. Gualdani, S. Mischler, C. Mouhot and C. Sparber, The Wigner-Fokker-Planck equation: stationary states and large time behavior, In progress.

5. J.-D. Deuschel and D. W. Stroock, J. Funct. Anal. 92, 30 (1990).

6. M. Ledoux, The concentration of measure phenomenon (Amer. Math. Soc., 2001).

7. D. Bakry and M. Émery, Propaganda for $\Gamma_{2}$, in From local times to global geometry, control and physics (Coventry, 1984/85), , Pitman Res. Notes Math. Ser. Vol. 150 (Longman Sci. Tech., Harlow, 1986) pp. 39-46. 